\title{
Aerosol Transmission of Aspergillus fumigatus in Cystic Fibrosis Patients in the Netherlands
}

\author{
Tobias G.P. Engel, Ellen Erren, \\ Koen S.J. Vanden Driessche, \\ Willem J.G. Melchers, Monique H. Reijers, \\ Peter Merkus, Paul E. Verweij
}

We collected sputum samples and cough plates from 15 cystic fibrosis patients in the Netherlands who were colonized with Aspergillus fumigatus; we recovered $A$. fumigatus of the same genotype in cough aerosols and sputum samples from 2 patients. The belief that transmission of A. fumigatus from cystic fibrosis patients does not occur should be reconsidered.

$\mathrm{P}$ rogressive lung injury in cystic fibrosis (CF) patients can lead to chronic colonization with bacteria and fungi $(1,2)$. Different routes of patient-to-patient transmission have been identified for various microorganisms (3). For saprophytic molds, such as Aspergillus fumigatus, exposure to aerosolized conidia in the environment is believed to be the primary route leading to colonization of the airways (3). Secretion of these fungi from the human lung into the environment is thought not to occur, and the general view is that humans are dead-end hosts of filamentous fungi. However, some reports have provided information suggesting that this belief might need to be revised (4). We set out to examine whether aerosol formation of A. fumigatus occurs in $\mathrm{CF}$ patients during coughing.

\section{The Study}

During 2017-2018, we invited 15 adult CF patients in the Netherlands who were colonized with A. fumigatus to participate in a cough plate experiment. We defined A. fumigatus colonization as the recovery of $A$. fumigatus from $>50 \%$ of sputum samples collected over the course of the 2 previous years. To enable genetic comparisons among study samples, we stored cultured fungal isolates from included patients in the Radboud University Medical Center Medical Microbiology Department (Nijmegen, the Netherlands)

Author affiliations: Radboud University Medical Center, Nijmegen, the Netherlands (T.G.P. Engel, E. Erren, K.S.J. Vanden Driessche, W.J.G. Melchers, M.H. Reijers, P. Merkus, P.E. Verweij); Center of Expertise in Mycology Radboud UMC/CWZ, Nijmegen (T.G.P. Engel, W.J.G. Melchers, M.H. Reijers, P.E. Verweij)

DOI: https://doi.org/10.3201/eid2504.181110 fungal species bank. We performed sample collection during 2 routine quarterly visits before or after routinely performed spirometry. We instructed participants to take a maximal inspiration and cough twice on each of 2 different agar plates, Sabouraud dextrose agar and Columbia blood agar, held at a $5-\mathrm{cm}$ distance from the participant's mouth. In addition, we collected a sputum sample on the same day or alternatively within a month if the participant was unable to produce sputum during the visit. We incubated Sabouraud dextrose agar cough plates at $28^{\circ} \mathrm{C}$ and Columbia blood agar cough plates at $36^{\circ} \mathrm{C}$ for 3 weeks and inspected daily for bacterial and fungal growth. We processed sputum samples according to CF guidelines (3). We collected individual CFUs (up to a maximum of 20 CFUs per sample) from cough plates and sputum cultures and genotyped A. fumigatus CFUs using microsatellite genotyping (5). We considered the detection of identical A. fumigatus genotypes from cough plates and sputum samples as proof of aerosolization of $A$. fumigatus from humans. We genotyped up to 6 sputum cultures of stored A. fumigatus isolates from each participant.

This study was reviewed by the institutional review board, which considered the study exempt from further institutional review board oversight in accordance with the law in the Netherlands on research with humans. All participants provided informed consent.

We cultured A. fumigatus from 18 (60\%) sputum samples collected from 11 different participants (Table). A. fumigatus was also recovered from $3(17 \%)$ of the 18 corresponding cough plate samples; these 3 samples were from 2 different participants. In both cases, cough plates had been acquired after the participant had undergone spirometry. Genotyping of the A. fumigatus isolates from sputum samples and cough plates from both participants showed identical genotypes. One participant was colonized with at least 10 different $A$. fumigatus genotypes (Figure). In this participant, the genotype recovered from the cough plate was not found in the sputum sample collected at the same visit but in the sputum sample recovered at the next visit. The second participant was colonized with a single genotype, which was recovered from multiple cough plates and sputum samples.

We also assessed for bacterial species present on cough plates and in sputum samples to enable comparison of transmission frequencies among pathogens. The cough 
Table. Characteristics of and growth of Aspergillus fumigatus, Pseudomonas aeruginosa, Staphylococcus aureus, and

Stenotrophomonas maltophilia in sputum samples and on cough plates from 15 cystic fibrosis patients, the Netherlands, 2017-2018*

\begin{tabular}{|c|c|c|c|c|c|c|c|c|c|c|c|c|}
\hline \multirow[b]{2}{*}{$\begin{array}{l}\text { Pt. } \\
\text { no. }\end{array}$} & \multirow[b]{2}{*}{$\begin{array}{l}\text { Age, } \\
\text { y/sex }\end{array}$} & \multirow{2}{*}{$\begin{array}{c}\text { FEV1, L } \\
(\% \\
\text { predicted) }\end{array}$} & \multirow{2}{*}{$\begin{array}{l}\text { Peak } \\
\text { flow, } \\
\text { L/s }\end{array}$} & \multirow[b]{2}{*}{$\begin{array}{c}\mathrm{AB} / \mathrm{AF} \\
\text { use }\end{array}$} & \multicolumn{4}{|c|}{ Growth on first/second cough plates } & \multicolumn{4}{|c|}{ Cultured from first/second sputum sample } \\
\hline & & & & & $\begin{array}{c}A . \\
\text { fumigatus }\end{array}$ & $\begin{array}{c}P . \\
\text { aeruginosa }\end{array}$ & $\begin{array}{c}S . \\
\text { aureus }\end{array}$ & $\begin{array}{c}S . \\
\text { maltophilia }\end{array}$ & $\begin{array}{c}A . \\
\text { fumigatus }\end{array}$ & $\begin{array}{c}P . \\
\text { aeruginosa }\end{array}$ & $\begin{array}{c}S . \\
\text { aureus }\end{array}$ & $\begin{array}{c}\text { S. } \\
\text { maltophilia }\end{array}$ \\
\hline 1 & $23 / F$ & $2.7(85)$ & 7.9 & Yes/no & $-1-$ & $+1-$ & $-1-$ & $-1-$ & $-1-$ & $+/+$ & $+/+$ & $+1-$ \\
\hline 2 & 20/F & $4.0(101)$ & 8.6 & Yes/no & $-1-$ & $-1-$ & $-1-$ & $-1+$ & $-/ N D$ & $-/ N D$ & $+/ \mathrm{ND}$ & $+/ \mathrm{ND}$ \\
\hline 3 & $22 / F$ & $2.3(74)$ & 7.9 & Yes/no & $-1-$ & $-1-$ & $-1+$ & $-1-$ & $+/+$ & $-1-$ & $+/+$ & $-1-$ \\
\hline 4 & 20/M & $3.2(61)$ & 7.4 & Yes/no & $+1-$ & $-1-$ & $-1-$ & $-1-$ & $+/+$ & $-1-$ & $+/+$ & $-1-$ \\
\hline 5 & $49 / F$ & $2.3(74)$ & 7.7 & No/no & $-1-$ & $-1-$ & $-1-$ & $-1-$ & $+1-$ & $-1-$ & $+/+$ & $-1-$ \\
\hline 6 & $43 / F$ & $2.7(82)$ & 7.2 & Yes/no & $-1-$ & $-1-$ & $-1-$ & $-1-$ & $+/+$ & $+/+$ & $-1-$ & $-1-$ \\
\hline 7 & $26 / \mathrm{M}$ & $4.6(106)$ & 10.9 & Yes/no & $-1-$ & $-1-$ & $+/+$ & $-1-$ & $+/ \mathrm{ND}$ & $-/ N D$ & $+/ \mathrm{ND}$ & $-/ N D$ \\
\hline 8 & $39 / F$ & $1.0(33)$ & 5.7 & Yes/no & $-1-$ & $-1-$ & $-1+$ & $-1-$ & $+/+$ & $-1-$ & $-1-$ & $+/+$ \\
\hline 9 & $49 / \mathrm{M}$ & $1.2(31)$ & 3.4 & Yes/no & $-1-$ & $+/+$ & $-1-$ & $-1-$ & $+/+$ & $+/+$ & $-1-$ & $-1-$ \\
\hline 10 & $22 / \mathrm{M}$ & $2.0(41)$ & 7.8 & Yes/no & $-1-$ & $+/+$ & $-1-$ & $-1-$ & $+1-$ & $+/+$ & $+/+$ & $-1-$ \\
\hline 11 & $58 / F$ & 2.2 (79) & 5.1 & Yes/no & $-1-$ & $-1-$ & $-1-$ & $-1-$ & $-1-$ & $+/+$ & $+1-$ & $-1-$ \\
\hline 12 & $31 / \mathrm{M}$ & $2.0(41)$ & 5.3 & Yes/no & $-1-$ & $+/+$ & $+/+$ & $-1-$ & $-1+$ & $-1+$ & $-1+$ & $-1-$ \\
\hline 13 & $51 / F$ & 0.7 (26) & 3.5 & Yes/no & $-1-$ & $+/+$ & $-1-$ & $-1-$ & $-1-$ & $+/+$ & $+/+$ & $-1-$ \\
\hline 14 & $30 / \mathrm{M}$ & $2.5(50)$ & 8.7 & Yes/no & $-1-$ & $+/+$ & $-1-$ & $-1-$ & $+/+$ & $+/+$ & $-1-$ & $-1+$ \\
\hline 15 & $27 / F$ & $3.8(102)$ & 8.4 & Yes/no & $+/+$ & $-1-$ & $-1-$ & $-1-$ & $+/+$ & $-1-$ & $-1-$ & $-1-$ \\
\hline
\end{tabular}

secretion frequency for Pseudomonas aeruginosa was 67\% $(10 / 15)$ and for Staphylococcus aureus was 19\% (3/16) (Table). No growth of Stenotrophomonas maltophilia on cough plates was observed.

Our study demonstrates that $A$. fumigatus can be recovered from cough aerosols from colonized CF patients with a similar frequency as $S$. aureus. This aerosolization is strikingly occurring in CF outpatients without any cavitary lesions or other serious complications. Cavitary lesions can facilitate sporulation of A. fumigatus inside patient lungs and thereby aids in fungal secretion.

By continuously monitoring indoor airborne fungal contamination with electrostatic dustfall collectors, Lemaire et al. identified patient airways as the source of
A. fumigatus contamination in an intensive care unit (4). Microsatellite genotyping showed that the airborne $\mathrm{A}$. $\mathrm{fu}$ migatus and isolates from the patient's respiratory samples were identical. That observation and our study results indicate that the current consensus that transmission of $A$. $f u$ migatus from colonized or infected patients does not occur should be reconsidered.

Although CF patients are typically colonized with unique $A$. fumigatus genotypes, several studies report the recovery of identical $A$. fumigatus genotypes from different $\mathrm{CF}$ patients (6). These genotypes could not be linked to a common environmental source and thus remained unexplained, but identical genotypes in different patients might point toward patient-to-patient transmission (6). Patient-derived
A

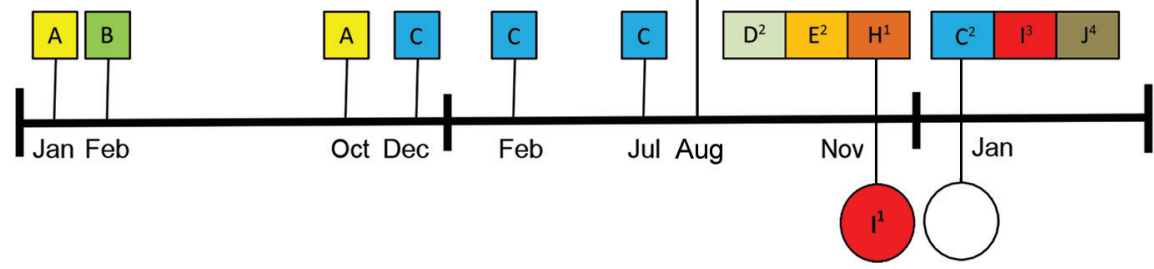

2016

2017

B
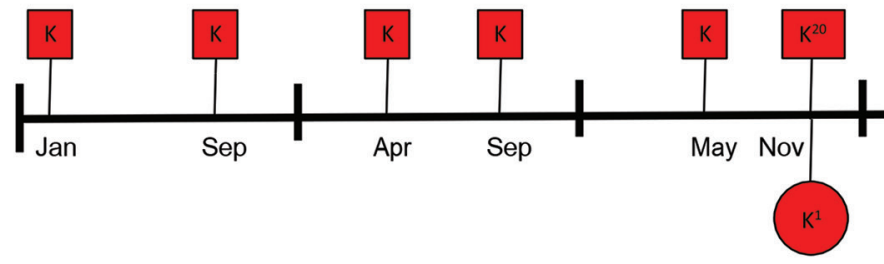

2017
Figure. Genotyping results of Aspergillus fumigatus isolates in sputum cultures and on cough plates obtained from 2 participants with cystic fibrosis demonstrating aerosol formation of $A$. fumigatus, the Netherlands, 2015-2018. For samples collected after August 2017 , a maximum of 20 isolates per sputum culture were saved. For samples collected earlier, only 1 sputum sample isolate was saved. Genotypes of the isolates collected from patient $4(\mathrm{~A})$ and $15(\mathrm{~B})$ are indicated. The superscript number indicates the number of isolates of that same genotype cultured from the collected sample. 
azole resistance mutations have been reported in CF patient (7) and environmental (8) isolates. The mechanism for acquisition of azole resistance is unknown for environmental isolates, but resistance might have developed in isolates during human infection or colonization and then subsequently spread into the environment. Our observation of A. fumigatus in cough aerosols suggests droplet or airborne transmission as potential routes of patient to patient transmission. Traits such as azole resistance might spread through cough aerosols from patient to patient or from patient to environment. Both participants with positive cough plates had undergone spirometry before coughing, indicating that maximal inspiration and expiration might facilitate the release of $A$. fumigatus.

Aerosolized A. fumigatus conidia from environmental sources could represent a greater and more continuous burden for $\mathrm{CF}$ patients than potentially aerosolized conidia from patients. However, some studies suggest that $A$. fumigatus in chronically infected patients undergoes an evolutionary trajectory resulting in strains with specific traits that are better adapted to the lung environment $(9,10)$. Endogenous and exogenous stress factors, such as host immunity or exposure to antifungal azoles, might result in the selection of traits that are better able to resist these stress factors (11). Isolates that are better adapted to the lung environment might have a greater propensity than environmental isolates to successfully colonize and persist in the airways of CF patients. In CF, $42 \%$ of azole resistance is derived through in-host selection and is thought to be associated with prolonged (prophylactic) use of anti-Aspergillus azoles $(12,13)$. Passaging through the lungs of CF patients conceivably could provide A. fumigatus isolates the opportunity to acquire specific traits that increase their ability to survive in different environments. Evolution experiments have confirmed the potential of A. fumigatus to rapidly adapt to various environments $(9)$.

\section{Conclusions}

In summary, our results show that A. fumigatus can be recovered from cough aerosols of colonized CF patients. These findings underscore the need for additional studies to further elaborate transmission dynamics of $A$. $f u$ migatus, evaluate if patient-to-patient transmission occurs, and determine if additional infection prevention measures are required.

\section{About the Author}

Mr. Engel is a resident in clinical microbiology and a doctoral candidate in the Radboud University Medical Center, Nijmegen, the Netherlands. His primary research interest is in mycology, especially fungal colonization in CF patients.

\section{References}

1. Elborn JS. Cystic fibrosis. Lancet. 2016;388:2519-31. http://dx.doi.org/10.1016/S0140-6736(16)00576-6

2. Schwarz C, Bouchara JP, Buzina W, Chrenkova V, Dmeńska H, de la Pedrosa EGG, et al. Organization of patient management and fungal epidemiology in cystic fibrosis. Mycopathologia. 2018;183:7-19. http://dx.doi.org/10.1007/s11046-017-0205-x

3. Saiman L, Siegel JD, LiPuma JJ, Brown RF, Bryson EA, Chambers MJ, et al.; Cystic Fibrous Foundation; Society for Healthcare Epidemiology of America. Infection prevention and control guideline for cystic fibrosis: 2013 update. Infect Control Hosp Epidemiol. 2014;35(Suppl 1):S1-67. http://dx.doi.org/ $10.1086 / 676882$

4. Lemaire B, Normand A-C, Forel J-M, Cassir N, Piarroux R, Ranque S. Hospitalized patient as source of Aspergillus fumigatus, 2015. Emerg Infect Dis. 2018;24:1524-7. http://dx.doi.org/10.3201/eid2408.171865

5. de Valk HA, Meis JF, Curfs IM, Muehlethaler K, Mouton JW, Klaassen $\mathrm{CH}$. Use of a novel panel of nine short tandem repeats for exact and high-resolution fingerprinting of Aspergillus fumigatus isolates. J Clin Microbiol. 2005;43:4112-20. http://dx.doi.org/10.1128/JCM.43.8.4112-4120.2005

6. de Valk HA, Klaassen CHW, Yntema JB, Hebestreit A, Seidler M, Haase G, et al. Molecular typing and colonization patterns of Aspergillus fumigatus in patients with cystic fibrosis. J Cyst Fibros. 2009;8:110-4. http://dx.doi.org/10.1016/j.jcf.2008.10.003

7. Morio F, Aubin GG, Danner-Boucher I, Haloun A, Sacchetto E, Garcia-Hermoso D, et al. High prevalence of triazole resistance in Aspergillus fumigatus, especially mediated by TR/L98H, in a French cohort of patients with cystic fibrosis. J Antimicrob Chemother. 2012;67:1870-3. http://dx.doi.org/10.1093/jac/dks160

8. Bader O, Tünnermann J, Dudakova A, Tangwattanachuleeporn M, Weig M, Groß U. Environmental isolates of azole-resistant Aspergillus fumigatus in Germany. Antimicrob Agents Chemother. 2015;59:4356-9. http://dx.doi.org/10.1128/AAC.00100-15

9. Verweij PE, Zhang J, Debets AJM, Meis JF, van de Veerdonk FL, Schoustra SE, et al. In-host adaptation and acquired triazole resistance in Aspergillus fumigatus: a dilemma for clinical management. Lancet Infect Dis. 2016;16:e251-60. http://dx.doi.org/10.1016/S1473-3099(16)30138-4

10. Ballard E, Melchers WJG, Zoll J, Brown AJP, Verweij PE, Warris A. In-host microevolution of Aspergillus fumigatus: a phenotypic and genotypic analysis. Fungal Genet Biol. 2018; 113:1-13. http://dx.doi.org/10.1016/j.fgb.2018.02.003

11. Zelante T, Iannitti RG, De Luca A, Arroyo J, Blanco N, Servillo G, et al. Sensing of mammalian IL-17A regulates fungal adaptation and virulence. Nat Commun. 2012;3:683. http://dx.doi.org/10.1038/ ncomms 1685

12. Mortensen KL, Jensen RH, Johansen HK, Skov M, Pressler T, Howard SJ, et al. Aspergillus species and other molds in respiratory samples from patients with cystic fibrosis: a laboratory-based study with focus on Aspergillus fumigatus azole resistance. J Clin Microbiol. 2011;49:2243-51. http://dx.doi.org/10.1128/JCM.00213-11

13. Hamprecht A, Morio F, Bader O, Le Pape P, Steinmann J, Dannaoui E. Azole resistance in Aspergillus fumigatus in patients with cystic fibrosis: a matter of concern? Mycopathologia. 2018;183:151-60. http://dx.doi.org/10.1007/s11046-017-0162-4

Address for correspondence: Tobias G.P. Engel, Radboud University Medical Center, Department of Medical Microbiology, Route 777, Geert Grooteplein Zuid 10, 6500 HB Nijmegen, the Netherlands; email: tobias.engel@radboudumc.nl 